\title{
Passive optimization of pump noise transfer function by narrow band-pass filtering in femtosecond fiber lasers
}

\author{
Peng Qin ${ }^{1}$, Sijia Wang ${ }^{1}$, Minglie $\mathrm{Hu}^{2}$, and Youjian Song ${ }^{2}$ \\ ${ }^{1}$ Qian Xuesen Laboratory of Space Technology, China Academy of Space Technology, Beijing 100094, China \\ ${ }^{2}$ Ultrafast Laser Laboratory, Key Laboratory of Opto-electronic Information Technical Science of Ministry of Education, \\ School of Precision Instruments and Opto-electronics Engineering, Tianjin University, Tianjin 300072, China \\ (Received 22 April 2019; revised 10 June 2019; accepted 27 June 2019)
}

\begin{abstract}
Fluctuation of pump power is one of the major sources of temporal and intensity noise in femtosecond fiber lasers. In this work, the transfer functions between the relative intensity noise (RIN) of the pump laser diode (LD) and the output RIN, between the RIN of the pump LD and timing jitter of femtosecond fiber lasers are systematically studied. It is demonstrated, for the first time to our knowledge, that the amplitude of the pump RIN transfer function can be effectively decreased by an intra-cavity narrow band-pass filter. In particular, for normal-dispersion lasers, the 3-dB bandwidth of the transfer function can also be narrowed by two-thirds, with a steeper falling edge. Furthermore, with the narrow band-pass filtering, the transfer function is almost independent of the net intra-cavity dispersion due to amplifier similariton formation. The proposed scheme can effectively isolate the pump-induced noise without the need of complex active pump LD control and intra-cavity dispersion management, thus providing an easy way for practical high-power, high-stability femtosecond fiber laser design and related high-precision applications outside the laboratory.
\end{abstract}

Keywords: band-pass filter; femtosecond fiber lasers; transfer function

\section{Introduction}

Mode-locked fiber lasers generate trains of femtosecond pulses with equal pulse width, intensity and timing interval, making them ideal sources in pump-probe experiments ${ }^{[1]}$, laser ablation ${ }^{[2]}$, timing synchronization ${ }^{[3]}$ and precise metrology ${ }^{[4]}$. To further extend their applications, various mode-locking mechanisms have been developed to support shorter pulse width and higher pulse energy ${ }^{[5-7]}$. In addition, a number of theoretical and experimental studies have been done to uncover the noise coupling mechanism as well as the noise suppression methods ${ }^{[8-11]}$. Besides amplified spontaneous emission (ASE) noise, which is known to be the main source of timing jitter ${ }^{[8,12]}$, pump power fluctuations also have significant effects on both amplitude and phase noise in femtosecond fiber lasers ${ }^{[13,14]}$. On one hand, pump power fluctuations would induce noise of the inversion level ${ }^{[15]}$, and thus increase the intensity noise of the pulse train ${ }^{[8,16]}$. On the other hand, it would affect the repetition rate and the carrier-envelope offset frequency through self-phase modulation, third-order dispersion and

Correspondence to: S. Wang, Qian Xuesen Laboratory of Space Technology, China Academy of Space Technology, Beijing 100094, China. Email: sj0607@163.com self-steepening ${ }^{[13,17]}$. In order to obtain high-energy pulses with ultra-high stability and uniformity, the noise induced by pump power fluctuations should be sufficiently suppressed.

Due to the limited lifetime of the gain medium, the mode-locked laser can function as a low-pass filter. The laser response to the pump laser diode (LD) intensity fluctuations has a bandwidth determined by the interplay of gain dynamics and laser dynamics ${ }^{[17]}$. For mode-locked fiber lasers, the lifetime of the gain medium is usually at the millisecond level ${ }^{[18]}$. The much faster laser dynamics can lead to a larger laser response bandwidth than that of the gain fiber. Previously, the influence of pump noise was mainly suppressed by feedback control. To push the locking bandwidth beyond the response limitation, phaselead compensation is always used ${ }^{[19,20]}$. Considering the dominant role of laser dynamics in the pump noise response of femtosecond fiber lasers, the transfer function between the pump and the output can be expected to be further optimized by improving the cavity design.

In this work, the transfer functions between the relative intensity noise (RIN) of the pump laser diode and the output RIN, between the RIN of the pump LD and timing jitter of femtosecond fiber lasers are systematically studied by inducing white noise modulation ${ }^{[21]}$ to the pump current 
under various intra-cavity net dispersion and spectral filtering conditions. It is demonstrated, for the first time to our knowledge, that the incorporation of a band-pass filter can effectively decrease the amplitude of the pump noise transfer function. In particular, for lasers with normal dispersion, the 3-dB bandwidth of the pump RIN transfer function could also be significantly narrowed by two-thirds, with a steeper falling edge. Furthermore, the transfer function in lasers with narrow band-pass filtering is almost independent of the net dispersion variation. The proposed scheme can effectively isolate the coupling between the pump power fluctuation and the output noise of femtosecond fiber lasers without the need for complex active pump LD control and intra-cavity dispersion management, thus demonstrating the potential for practical high-power, high-stability laser design and related high-precision applications outside the laboratory.

\section{Experimental setup and results}

\subsection{Characterization of the transfer function between the RIN of the pump and the laser}

A home-made nonlinear polarization evolution (NPE) modelocked fiber laser is used as the laser under test (LUT), as shown in Figure 1. The fiber part consists of a segment of Yb-doped gain fiber and the pigtail of a wavelength-division multiplexer (WDM) and collimators. The lengths of Yb-fiber and passive fiber are $23 \mathrm{~cm}$ and $51 \mathrm{~cm}$, respectively, and the total dispersion induced by the fiber part is approximately $+0.018 \mathrm{ps}^{2}$. The intra-cavity net dispersion can be adjusted by changing the separation of the diffraction grating pair, and the repetition rate of the LUT is fixed at $160 \mathrm{MHz}$ by adjusting the free space optical path accordingly. The laser is pumped by a grating-stabilized $\mathrm{LD}$, and $5 \%$ of the pump power is tapped out to monitor its fluctuations. The LUT can be mode-locked in the passive self-similar, stretched pulse and soliton regimes when the net dispersion is set to normal, close to zero and anomalous, respectively. An additional 7.5-nm band-pass filter can be inserted before the input collimator to achieve amplifier similariton mode-locking. The RIN of the LD and the LUT in different mode-locking regimes is detected by a low-noise amplified detector. For a $1 \mathrm{M} \Omega$ load, the average voltage of the detector output is fixed at $5 \mathrm{~V}$ by adjusting the tunable attenuator (TA) so as to set the power of the incident optical signal in the linear range of the detector. Then the detected signal is analyzed by a fast Fourier transform spectrum analyzer in the frequency range from $1 \mathrm{~Hz}$ to $100 \mathrm{kHz}$.

For femtosecond fiber lasers, the RIN of laser output is usually lower than that of the pumping LD, which could be easily covered by the noise floor of the detection system ${ }^{[22]}$. As a result, the measurement of the transfer function between the RIN of the LD and the laser is always done by inducing additional modulation ${ }^{[17,23]}$. In this work, white noise

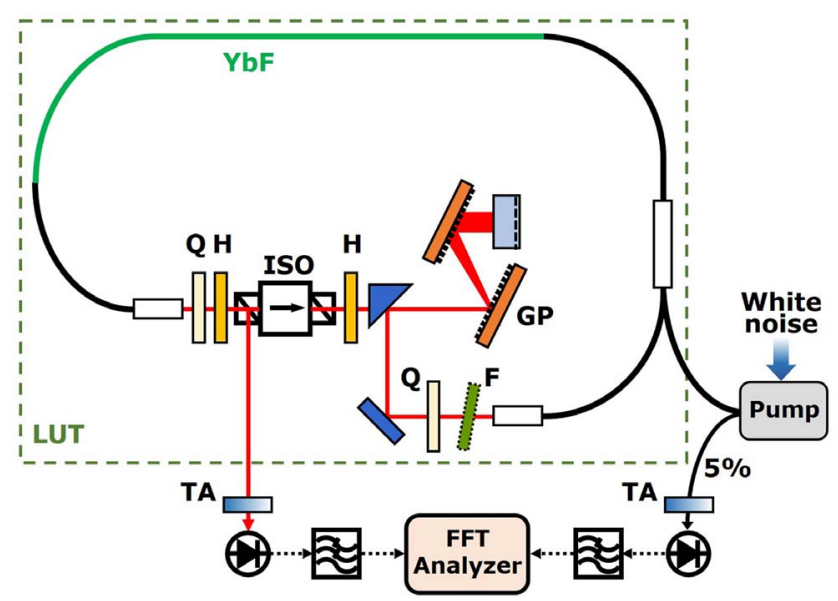

Figure 1. Configuration of the RIN transfer function characterization system. YbF, ytterbium-doped fiber; Q, quarter waveplate; H, half waveplate; ISO, isolator; F, band-pass filter; GP, grating pair; LUT, laser under test; TA, tunable attenuator.

modulation in the frequency range below $100 \mathrm{kHz}$ is used. To characterize the transfer function, the RIN data of the LUT and the LD are firstly measured without any modulation, and recorded as $R_{0}$ and $R_{\mathrm{LD} 0}$, respectively. Secondly, the white noise signal is applied to the LD controller. By increasing the amplitude of modulation, the RIN levels of both the LUT and LD could be totally separated from those without modulation, and the RIN data are recorded as $R_{1}$ and $R_{\mathrm{LD} 1}$, respectively. Since the white noise modulation only introduces incoherent components to the pump power fluctuation, the transfer function of RIN can be calculated as

$$
T_{\mathrm{RIN}}=\frac{R_{1}-R_{0}}{R_{\mathrm{LD} 1}-R_{\mathrm{LD} 0}}
$$

First, the transfer function of the RIN in a stretched pulse mode-locked fiber laser is measured with different modulation amplitudes. The net dispersion is $-0.001 \mathrm{ps}^{2}$, and the pump power coupled into the laser cavity is $240 \mathrm{~mW}$. The peak-to-peak amplitude of the pump power fluctuations is set to $3 \mathrm{~mW}$ and $6 \mathrm{~mW}$, respectively and the RIN data are summarized in Figures 2(a) and 2(b). It can be seen that even $3-\mathrm{mW}$ power fluctuation, which corresponds to $1.25 \%$ of the total pump power, could lead to a significant rise in the low-frequency RIN of the laser output. At the same time, the high-frequency RIN level rolls off exponentially as in a low-pass filter. The 3-dB bandwidth of the RIN curve is approximately $2 \mathrm{kHz}$, which is mainly determined by the gain relaxation time and the laser dynamics ${ }^{[17]}$. By further increasing the modulation amplitude, the RIN level of the LUT shows an overall rise in the full frequency range, while the shape of the RIN curve remains the same. The calculated transfer function curves are plotted in Figure 2(c). Although the RIN levels vary by approximately $6 \mathrm{~dB}$, the transfer function curves with different modulation depths match very well with each other. 

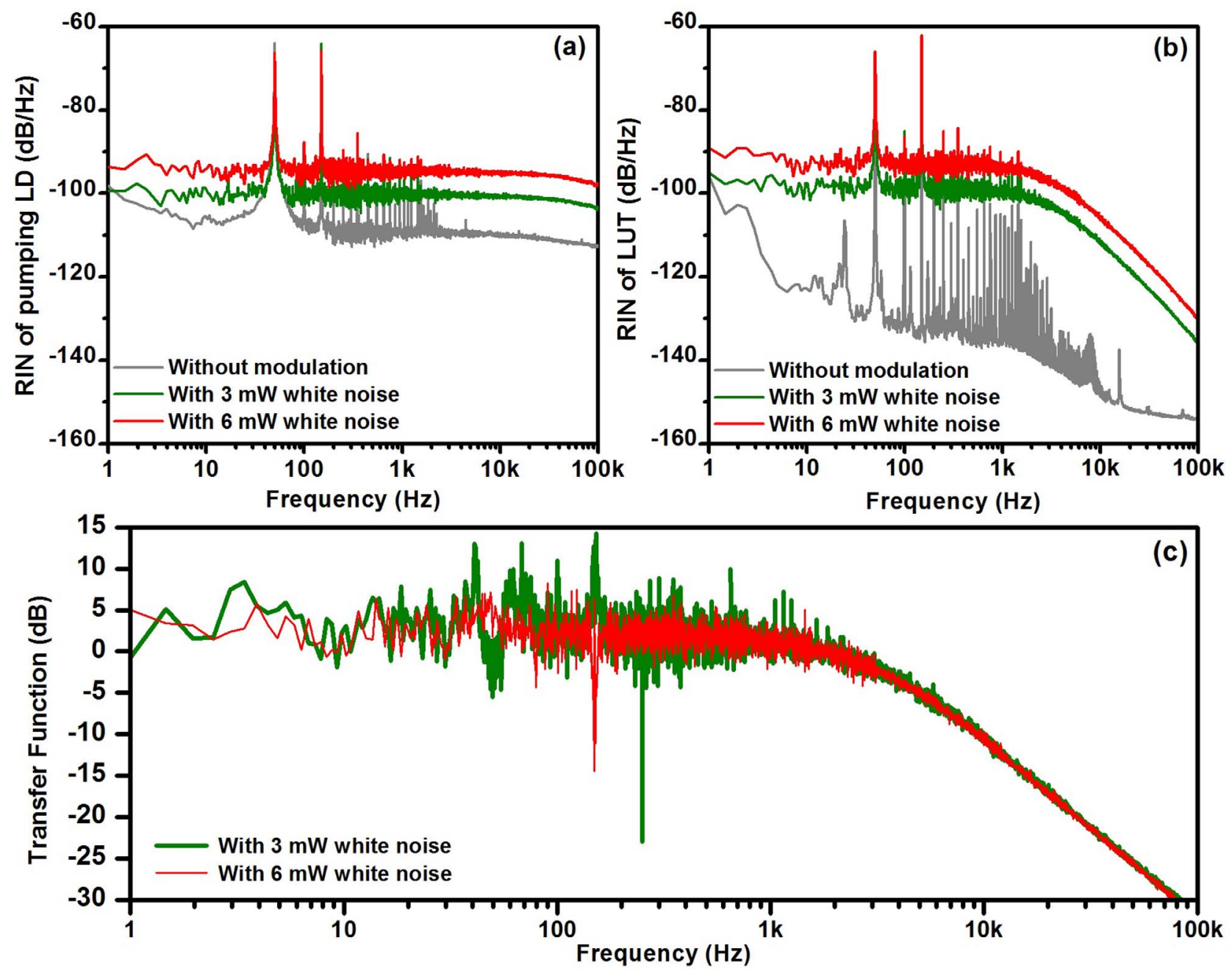

Figure 2. (a) RIN of the pump LD with (red and green) and without (gray) white noise modulation. (b) RIN of the LUT with (red and green) and without (gray) white noise modulation. (c) Transfer function of the RIN measured by applying $3 \mathrm{~mW}$ (green) and $6 \mathrm{~mW}$ (red) white noise modulation.

Second, the transfer function of the RIN is systematically studied under a variety of net dispersions. In the experiments, for the laser without the band-pass filter, the mode-locking regime varies with the net dispersion. The pump power is adjusted to be $300 \mathrm{~mW}, 360 \mathrm{~mW}, 240 \mathrm{~mW}$ and $200 \mathrm{~mW}$ at $+0.004 \mathrm{ps}^{2},+0.007 \mathrm{ps}^{2},-0.001 \mathrm{ps}^{2}$ and $-0.012 \mathrm{ps}^{2}$, respectively. When the net dispersion is normal, the LUT without intra-cavity filtering works in the passive self-similar regime. As shown by the black curve in Figure 3(a), the transfer function of the RIN exhibits lowpass-filter features. The $3-\mathrm{dB}$ bandwidth is $18 \mathrm{kHz}$, and the amplitude of the passband is $-1.2 \mathrm{~dB}$. As the net dispersion becomes larger, the roll-off slope becomes flatter, and the amplitude of the passband becomes higher, as shown by the blue curve in Figure 3(b). When the net dispersion is set to $-0.001 \mathrm{ps}^{2}$, the LUT without the band-pass filter is mode-locked in the stretched pulse regime. As shown by the green curve in Figure 3(c), the amplitude of the transfer function is $1.5 \mathrm{~dB}$, and the $3-\mathrm{dB}$ bandwidth is $2 \mathrm{kHz}$, which is close to the lower limit set by the upper-state lifetime of the Er-doped fiber ${ }^{[17]}$. By further increasing the net dispersion to $-0.012 \mathrm{ps}^{2}$, the LUT is mode-locked in the soliton regime. As shown by the purple curve in Figure 3(d), the $3-\mathrm{dB}$ bandwidth is broadened to $6 \mathrm{kHz}$, and the amplitude is also higher than that at $-0.001 \mathrm{ps}^{2}$. The optical spectra in each mode-locking regime are plotted in the corresponding insets of Figure 3. The corresponding full-width at halfmaximum (FWHM) durations of the compressed pulses are $89 \mathrm{fs}, 126 \mathrm{fs}, 41 \mathrm{fs}$ and $113 \mathrm{fs}$ at $+0.004 \mathrm{ps}^{2},+0.007 \mathrm{ps}^{2}$, $-0.001 \mathrm{ps}^{2}$ and $-0.012 \mathrm{ps}^{2}$, respectively. The temporal pulse profiles vary significantly with the net dispersion of laser. It can be seen that the amplitude and bandwidth of the transfer function vary significantly with the dispersion map. As the absolute value of net dispersion increases, the intensity noise coupling between the pump and fiber laser would always become stronger. In order to block the influence of the pump power fluctuations, optimization of the dispersion map and mode-locking regime is indispensable. 

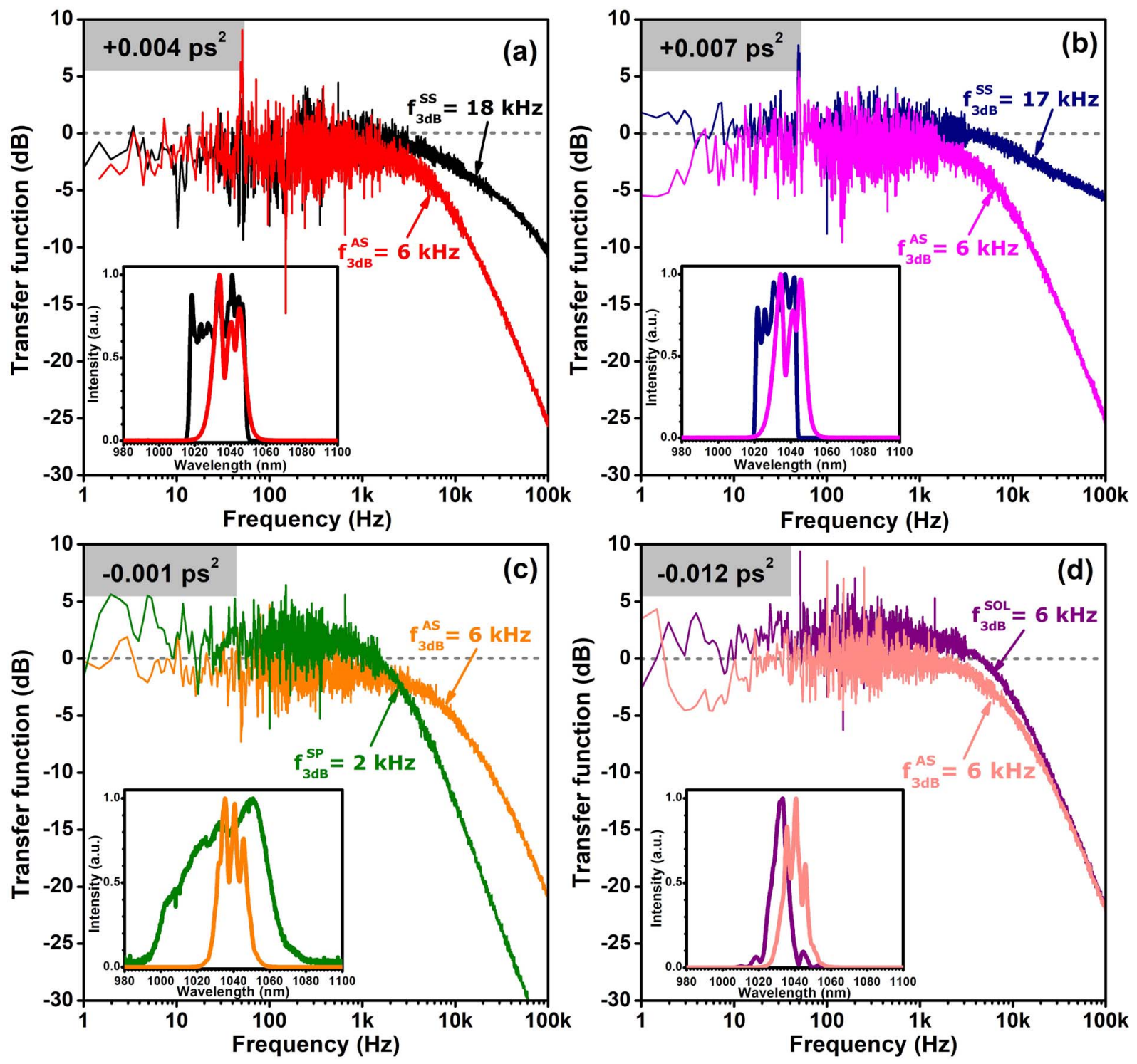

Figure 3. Transfer function curves of the RIN at (a) $+0.004 \mathrm{ps}^{2}$, with (red) and without (black) filter; (b) $+0.007 \mathrm{ps}^{2}$, with (pink) and without (blue) filter; (c) $-0.001 \mathrm{ps}^{2}$, with (orange) and without (green) filter; (d) $-0.012 \mathrm{ps}^{2}$, with (salmon) and without (purple) filter. SS, passive self-similar; AS, amplifier similariton; SP, stretched pulse; SOL, soliton. Insets: optical spectra of corresponding mode-locking regimes.

When the narrow band-pass filter is inserted, the modelocking regime of the LUT is switched to amplifier similariton, and the optical spectrum almost remains the same, even though the intra-cavity dispersion varies from $+0.007 \mathrm{ps}^{2}$ to $-0.012 \mathrm{ps}^{2}$. The corresponding FWHM durations exhibit similar values of $102 \mathrm{fs}, 106 \mathrm{fs}, 110 \mathrm{fs}$ and $118 \mathrm{fs}$ at $+0.004 \mathrm{ps}^{2},+0.007 \mathrm{ps}^{2},-0.001 \mathrm{ps}^{2}$ and $-0.012 \mathrm{ps}^{2}$, respectively. Apparently, the incorporation of a band-pass filter has indeed changed the temporal profiles of the pulses. The pump power is set to be $360 \mathrm{~mW}$ and $300 \mathrm{~mW}$ over the normal-dispersion region $\left(+0.004 \mathrm{ps}^{2}\right.$ and $+0.007 \mathrm{ps}^{2}$ ) and the anomalous dispersion region $\left(-0.001 \mathrm{ps}^{2}\right.$ and $\left.-0.012 \mathrm{ps}^{2}\right)$, respectively. The transfer function curves corresponding to the aforementioned net dispersion conditions are plotted in Figures 3(a)-3(d), respectively. When the net dispersion is normal, the lowpass feature in the gain fiber is significantly strengthened via insertion of the band-pass filter. As shown in Figures 3(a) and $3(\mathrm{~b})$, the $3-\mathrm{dB}$ bandwidth of the transfer function is narrowed by two-thirds to $6 \mathrm{kHz}$, and the roll-off slope also becomes much steeper than that without the filter. As a result, the intensity noise coupled from the pump fluctuation is further isolated in the high-frequency regime, which gives a reasonable explanation for the RIN reduction in normaldispersion femtosecond fiber lasers via intra-cavity bandpass filtering in Ref. [24]. When the net dispersion is anomalous, the intensity noise coupling in the low-frequency range is significantly suppressed. As shown in Figures 3(c) 


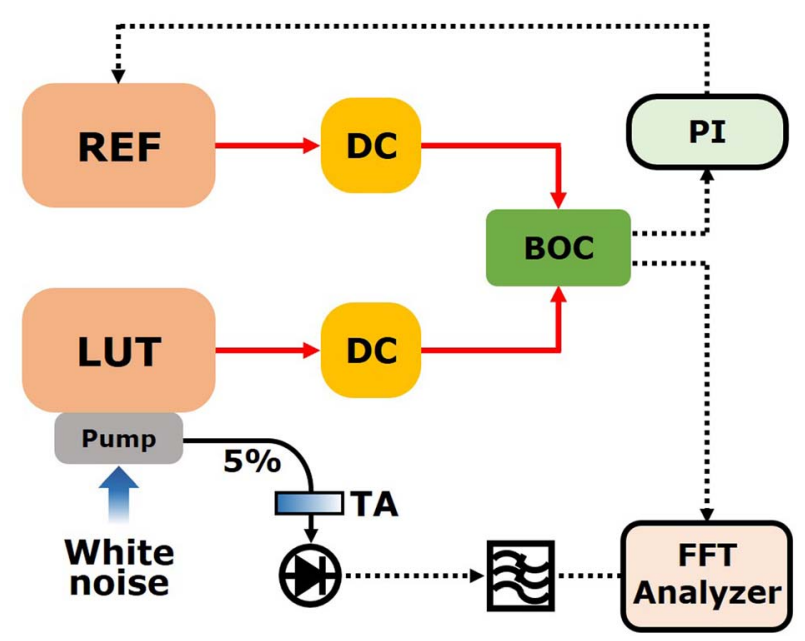

Figure 4. Configuration of the timing jitter transfer function characterization system. REF, reference laser; DC, dispersion compensation system; BOC, balanced optical cross-correlation system; PI, proportional-integral controller.

and 3(d), the amplitude of the transfer function in the filtered conditions is decreased by $2.8 \mathrm{~dB}$ and $2.2 \mathrm{~dB}$ from that without the filter, respectively. Furthermore, upon insertion of the band-pass filter, the amplitude of the transfer function is always restricted below $0 \mathrm{~dB}$, despite the net dispersion variation. In the meantime, the $3-\mathrm{dB}$ bandwidth is fixed at $6 \mathrm{kHz}$, and the roll-off slope almost remains the same. In this way, the RIN coupled from the pump power fluctuation is sufficiently suppressed in the whole frequency range by simply inserting a spectral filter. Although the 3-dB bandwidth is broader than that in the stretched pulse regime, complex optimization of net dispersion and mode-locking condition is not necessary, thus providing an easier way to eliminate the impact of instability of the pump.

\subsection{Characterization of transfer function between the RIN of the pump and the timing jitter of the laser}

In order to characterize the transfer function between the RIN of the pump LD and the timing jitter of the laser output, another femtosecond fiber laser with nearly identical structure is built as the reference (REF). The laser REF works in the stretched pulse regime with its net dispersion fixed at $0 \mathrm{ps}^{2}$. As shown in Figure 4, the timing jitter of the LUT is measured with the balanced optical cross-correlation (BOC) method when the repetition rates of the two lasers are synchronized with a proportional-integral (PI) controller ${ }^{[9]}$. To characterize the free-running jitter level in a broader frequency range, the locking bandwidth is kept below $1 \mathrm{kHz}$ by optimizing the phase-locking parameters. On the other hand, only 3-mW white noise modulation is applied to the pump LD of REF, so as not to break the repetition-rate synchronization.
The timing jitter data with and without modulation are recorded as $J_{1}$ and $J_{0}$, respectively, and the transfer function between the RIN of the LD and the timing jitter of the LUT can be calculated as

$$
T_{\mathrm{jitter}}=\frac{J_{1}-J_{0}}{R_{\mathrm{LD} 1}-R_{\mathrm{LD} 0}}
$$

First, the transfer function of the LUT is measured when the net dispersion is set to $-0.001 \mathrm{ps}^{2}$. The mode-locking regime of the LUT can be set to amplifier similariton or stretched pulse by inserting or removing the 7.5-nm bandpass filter, respectively. As shown in Figure 5(a), both curves exhibit the low-pass features. When the LUT is filter-free, the $3-\mathrm{dB}$ bandwidth is $5 \mathrm{kHz}$, and the passband amplitude is approximately $84.31 \mathrm{~dB}$. Upon insertion of the filter, the amplitude is decreased to $79.9 \mathrm{~dB}$, while the 3 - $\mathrm{dB}$ bandwidth is broadened to $23 \mathrm{kHz}$. Such changes are similar to that shown in the transfer function of the RIN, as shown in Figure 3(c).

Second, the net dispersion of the LUT is set to $+0.007 \mathrm{ps}^{2}$ and $+0.004 \mathrm{ps}^{2}$, respectively. When the LUT is filterfree, it is mode-locked in the passive self-similar regime, whose ASE-induced timing jitter is always much higher than that of the stretched pulse regime ${ }^{[25]}$. As a result, the repetition-rate synchronization of the two lasers could be easily broken by the addition of white noise modulation, leading to failure of the transfer function measurement. When the band-pass filter is inserted, the mode-locking regime is switched to amplifier similariton. Since the ASEinduced timing jitter is suppressed by the band-pass filter, the transfer function between the RIN of the LD and the timing jitter of the laser output can be characterized with the aforementioned method. As shown in Figure 5(b), the transfer functions of filtered conditions are similar to each other, although the net dispersion varies from $-0.001 \mathrm{ps}^{2}$ to $+0.007 \mathrm{ps}^{2}$. The nearly-constant transfer function results from the similar nonlinear loss factor, which is mainly decided by the intra-cavity dynamics ${ }^{[17]}$. Upon insertion of the band-pass filter, the pulse evolution pattern is restricted to amplifier similariton, which counteracts the influence of the variation of the net dispersion.

\section{Conclusion}

In conclusion, the transfer function between the RIN of the pump LD and the output noise of femtosecond fiber lasers is systematically characterized in terms of intra-cavity net dispersion and band-pass filtering conditions. Upon insertion of the spectral filter, the bandwidth of the RIN transfer function is significantly narrowed in normal-dispersion lasers. In fiber lasers with anomalous dispersion, the amplitude of the RIN transfer function is decreased. Furthermore, the RIN transfer function in filtered fiber lasers is almost invariant with the 

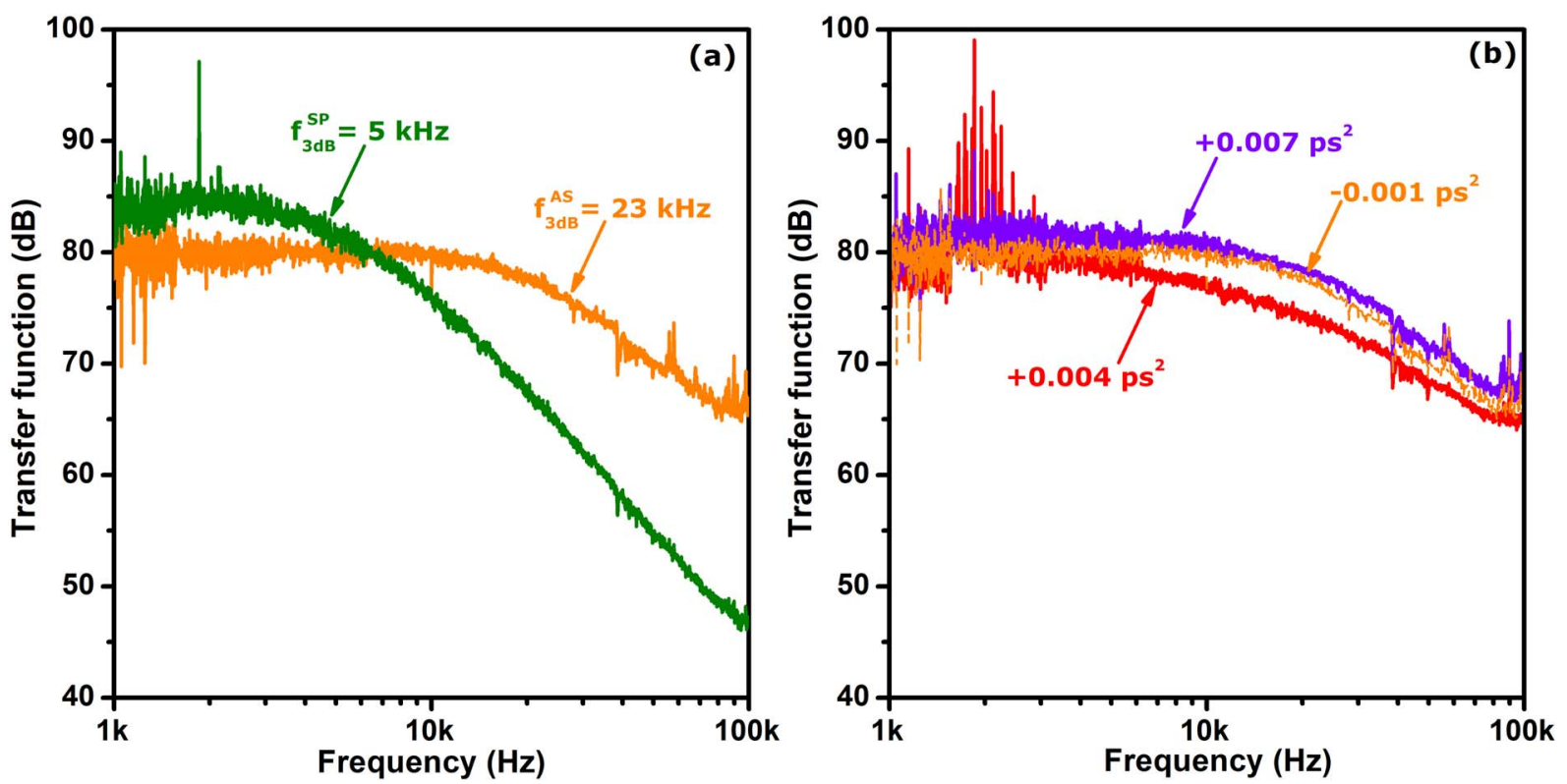

Figure 5. Transfer function curves of the timing jitter (a) at $-0.001 \mathrm{ps}^{2}$, with (orange) and without (green) filter and (b) for various levels of dispersion with filter.

net dispersion map, while its amplitude is always restricted below $0 \mathrm{~dB}$. The transfer function between the RIN of the $\mathrm{LD}$ and the timing jitter is also constant on inserting the narrow band-pass filter, despite the net dispersion variation. Although the bandwidth of the transfer function in filtered lasers is broader than in stretched pulse lasers, the amplitude in the low-frequency domain is sufficiently decreased. The study here provides a simple and effective way to suppress the amplitude and phase noise coupled from the fluctuation of pump power without complex active control and intra-cavity dispersion management, thus demonstrating the potential for high-precision real-world applications of femtosecond fiber lasers.

\section{Acknowledgements}

This work was funded by the National Science and Technology Innovation Special Zone Program of China, Independent Innovation Project of China Academy of Space Technology, Science and Technology Innovation Research and Development Project of China Aerospace Science and Technology Corporation, and National Natural Science Foundation of China (No. 61675150).

\section{References}

1. M. Domke, S. Rapp, M. Schmidt, and H. P. Huber, Opt. Express 20, 10330 (2012).

2. C. Kerse, H. Kalaycioğlu, P. Elahi, B. Çetin, D. K. Kesim, Ö. Akçaalan, S. Yavaş, M. D. Aşık, B. Öktem, H. Hoogland, R. Holzwarth, and F. Ö. Ilday, Nature 537, 84 (2016).
3. M. Xin, K. Safak, M. Y. Peng, A. Kalaydzhyan, W.-T. Wang, O. D. Mucke, and F. X. Kartner, Light: Sci. Appl. 6, e16187 (2017).

4. J. Lee, Y.-J. Kim, K. Lee, S. Lee, and S.-W. Kim, Nat. Photon. 4, 716 (2010)

5. K. Tamura, E. P. Ippen, H. A. Haus, and L. E. Nelson, Opt. Lett. 18, 1080 (1993).

6. A. Chong, J. Buckley, W. Renninger, and F. Wise, Opt. Express 14, 10095 (2006).

7. W. H. Renninger, A. Chong, and F. W. Wise, Opt. Express 19, 22496 (2011).

8. H. A. Haus and A. Mecozzi, IEEE J. Quantum Electron. 29, 983 (1993)

9. Y.-J. Song, K. Jung, and J. Kim, Opt. Lett. 36, 1761 (2011).

10. P. Qin, S.-J. Wang, M.-L. Hu, and Y.-J. Song, IEEE Photon. J. 10, 1500208 (2018).

11. Y. Wang, H.-C. Tian, D. Hou, F. Meng, Y.-X. Ma, H. Xu, F. X. Kärtner, Y.-J. Song, and Z.-G. Zhang, Opt. Express 27, 11273 (2019).

12. S. Namiki and H. A. Haus, IEEE J. Quantum Electron. 33, 649 (1997).

13. J. J. McFerran, W. C. Swann, B. R. Washburn, and N. R. Newbury, Opt. Lett. 31, 1997 (2006).

14. K. Wu, P. P. Shum, S. Aditya, C.-M. Ouyang, J. H. Wong, H. Q. Lam, and K. E. K. Lee, Opt. Lett. 37, 1901 (2012).

15. R. Paschotta, Appl. Phys. B 79, 163 (2004).

16. K. Wu, J. H. Wong, P. Shum, S. Fu, C.-M. Ouyang, H.-H. Wang, E. J. R. Kelleher, A. I. Chernov, E. D. Obraztsova, and J.-P. Chen, Opt. Express 18, 16663 (2010).

17. B. R. Washburn, W. C. Swann, and N. R. Newbury, Opt. Express 13, 10622 (2005).

18. R. Paschotta, J. Nilsson, P. R. Barber, J. E. Caplen, A. C. Tropper, and D. C. Hanna, Opt. Commun. 136, 375 (1997).

19. J. J. McFerran, W. C. Swann, B. R. Washburn, and N. R. Newbury, Appl. Phys. B 86, 219 (2007).

20. N. R. Newbury and W. C. Swann, J. Opt. Soc. Am. B 24, 1756 (2007).

21. P. Z. Marmarelis and K.-I. Naka, Science 175, 1276 (1972). 
22. J. Chen, J. W. Sickler, E. P. Ippen, and F. X. Kärtner, Opt. Lett. 32, 1566 (2007).

23. A. J. Benedick, J. G. Fujimoto, and F. X. Kartner, Nat. Photon. 6, 97 (2012).
24. P. Qin, Y.-J. Song, H. Kim, J. Shin, D. Kwon, M.-L. Hu, C.-Y. Wang, and J. Kim, Opt. Express 22, 28276 (2014).

25. Y.-J. Song, C. Kim, K. Jung, H. Kim, and J. Kim, Opt. Express 19, 14518 (2011) 